\title{
A Metabonomic Comparison of Urinary Changes in Zucker and GK Rats
}

\author{
Liang-Cai Zhao, ${ }^{1}$ Xiao-Dong Zhang, ${ }^{1}$ Shi-Xian Liao, ${ }^{2}$ Hong-Chang Gao, ${ }^{2}$ \\ He-Yao Wang, ${ }^{1}$ and Dong-Hai Lin ${ }^{1,3}$
}

${ }^{1}$ Shanghai Institute of Materia Medica, The Chinese Academy of Sciences, Shanghai 201203, China

${ }^{2}$ School of Pharmacy, Wenzhou Medical College, Wenzhou 325035, China

${ }^{3}$ College of Chemistry and Chemical Engineering, Xiamen University, Xiamen 361005, China

Correspondence should be addressed to Hong-Chang Gao, gaohc27@gmail.com and Dong-Hai Lin, dhlin@mail.shcnc.ac.cn

Received 29 June 2010; Accepted 14 September 2010

Academic Editor: Ronald Tjeerdema

Copyright ( $\odot 2010$ Liang-Cai Zhao et al. This is an open access article distributed under the Creative Commons Attribution License, which permits unrestricted use, distribution, and reproduction in any medium, provided the original work is properly cited.

To further investigate pathogenesis and pathogenic process of type 2 diabetes mellitus (T2DM), we compared the urinary metabolic profiling of Zucker obese and Goto-kakizaki (GK) rats by NMR-based metabonomics. Principal component analysis (PCA) on urine samples of both models rats indicates markedly elevated levels of creatine/creatinine, dimethylamine, and acetoacetate, with concomitantly declined levels of citrate, 2-ketoglurarate, lactate, hippurate, and succinate compared with control rats, respectively. Simultaneously, compared with Zucker obese rats, the GK rats show decreased levels of trimethylamine, acetate, and choline, as well as increased levels of creatine/creatinine, acetoacetate, alanine, citrate, 2-ketoglutarate, succinate, lactate, and hippurate. This study demonstrates metabolic similarities between the two stages of T2DM, including reduced tricarboxylic acid (TCA) cycle and increased ketone bodies production. In addition, compared with Zucker obese rats, the GK rats have enhanced concentration of energy metabolites, which indicates energy metabolic changes produced in hyperglycemia stage more than in insulin resistance stage.

\section{Introduction}

Diabetes mellitus is the most common metabolic disease in the world. Over 90 percent of patients with diabetes belong to type 2 diabetes (T2DM) [1]. T2DM is a metabolic disorder characterized by a chronic hyperglycemia with disturbances of carbohydrate, fat, and protein metabolism resulting from defects in insulin secretion or insulin action [2]. Although the pathogenesis of the disease remains unclear, insulin resistance plays an important role in its pathogenic process, which precedes the clinical development of diabetes by 10 20 years $[3,4]$. However, little is known about what this inherited metabolic relation between the two stages.

In order to give new insight into diabetic process itself, as well as conduct clinical instruction, it is necessary to clarify the global metabolic alteration that characterizes its progression. Metabonomics, defined as "the quantitative measurement of the dynamic multiparametric metabolic response of living systems to pathophysiological stimuli or genetic modification" [5], is increasingly being applied to the study of disease, especially in metabolic disease [6]. With this technology Williams and his colleagues detected differences between plasma $[7,8]$ and urinary $[9,10]$ metabolic profiles of Zucker obese and control rats. The metabolic changes included the decreased level of taurine, together with changes in the relative proportion of tricarboxylic acid (TCA) cycle. Salek et al. [11] compared metabolic similarities among different type 2 diabetic models (rat and mouse) and human sufferers and identified perturbations in metabolism common to all three species, except changes of TCA cycle, perturbations in nucleotide metabolism were also determined. Wijekoon et al. [12] compared metabolic changes of amino acids in two stages of Zucker diabetic rat: prediabetic insulin-resistant and hyperglycemia stage. They found that concentrations of many gluconeogenic amino acids were lower at both stages whereas the levels of the branchedchain amino acids were elevated. Although a number of 


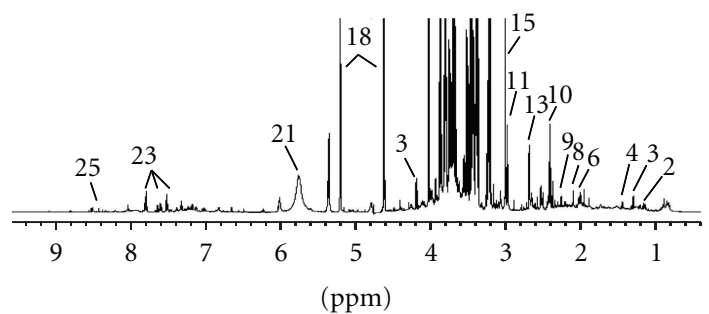

(a)

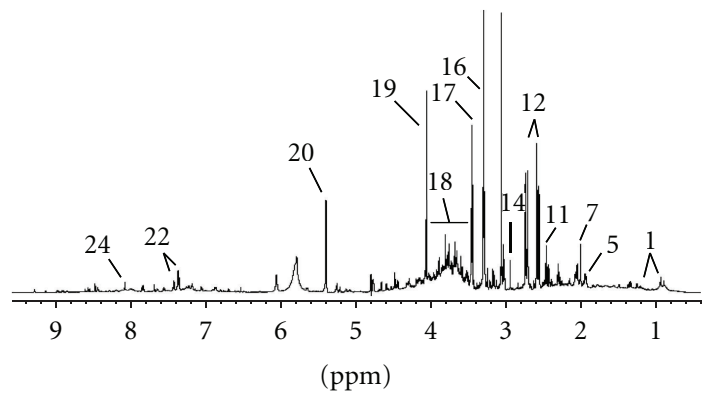

(b)

Figure 1: ${ }^{1} \mathrm{H}$ NMR spectra of urinary samples obtained from the GK rat (a) and Zucker obese rat (b), respectively. (1) leucine + isoleucine; (2) $\beta$-hydroxybutyrate; (3) lactate; (4) alanine; (5) acetate; (6) glutamate; (7) N-acetylglycoprotein; (8) methonine; (9) acetoacetate; (10) succinate; (11) 2-ketoglutarate; (12) citrate; (13) dimethylamine; (14) trimethylamine; (15) creatine + creatinine; (16) taurine; (17) taurine; (18) glucose; (19) creatinine; (20) allantoin; (21) urea; (22) phenylalanine; (23) hippurate; (24) Nmethylnicotinamide; (25) formate.

diabetes-related studies have been reported in recent years, the similarities and differences of metabolic changes between stages of insulin resistance and T2DM remain unclear. To further understand the pathogenic process of T2DM and identify its characteristics, we chose two relevant rat models: the Zucker obese rat and Goto-kakizaki (GK) rat, which represent two pathogenetic processes of T2DM, respectively: insulin resistance and hyperglycemia stage. Then in the present study, the urinary metabolic profiles of Zucker obese rats and GK rats were compared by NMR-based metabonomics, and we aimed to find the relation between the two diabetic stages.

\section{Materials and Methods}

2.1. Subjects. Spontaneously diabetic male GK rats (Shanghai Laboratory Animal Co., Ltd. Shanghai, China; 60 days of age; $n=8$ ) weighed $263.0 \pm 5.5 \mathrm{~g}$ at the beginning of experiments. The corresponding control animals were male Wistar rats with similar age. Male Zucker obese rats $(f a / f a$; weighed $421.3 \pm 10.5 \mathrm{~g} ; n=5$ ) and their lean littermates $(\mathrm{fa} / \mathrm{+})$, aged 8 weeks, were obtained from the Institute of Laboratory Animals, Kyoto University, and maintained at the Shanghai Institute of Materia Medica, Chinese Academy of Sciences.

Animals were kept in a barrier system with regulated temperature and humidity and on a 12/12h light-dark cycle with lights on at 08:00 AM. During the whole experimental process, rats were fed with certified standard rat chow and tap water ad libitum. All animal treatments were strictly in accordance with the National Institutes of Health Guide for the Care and Use of Laboratory Animals.

2.2. Collection and Preparation of Samples. Urinary samples were collected, for 24 hours, each of the Zucker and GK rats in individual urine collection cages over ice into $0.1 \mathrm{~mL}$ of $1 \%$ sodium azide solution. The urine samples were centrifuged, and the supernatant was stored at $-80^{\circ} \mathrm{C}$ before analysis. Prior to NMR analysis, urine samples were thawed, and $300 \mu \mathrm{L}$ of aliquots were mixed with $300 \mu \mathrm{L}$ of phosphate buffer $\left(0.2 \mathrm{~mol} / \mathrm{L} \mathrm{Na} \mathrm{HPO}_{4} / \mathrm{NaH}_{2} \mathrm{PO}_{4}, \mathrm{pH} 7.4\right)$ to minimize variations in $\mathrm{pH}$. The samples were centrifuged at $12000 \mathrm{~g}$ for 10 minutes at $4^{\circ} \mathrm{C}$ to separate any precipitate. Aliquots of $500 \mu \mathrm{L}$ of the resulting supernatants were moved into $5 \mathrm{~mm}$ NMR tubes containing $100 \mu \mathrm{L}$ solution of $2,2^{\prime}, 3,3^{\prime}$ deuterotrimethylsilylproprionic acid (TSP) in $\mathrm{D}_{2} \mathrm{O}$ (the final concentration is $1.5 \mathrm{mmol} / \mathrm{L}) . \mathrm{D}_{2} \mathrm{O}$ provided a field frequency lock. TSP was used as the chemical shift reference.

2.3. ${ }^{1}$ H NMR Spectroscopic Analysis of Samples. All NMR spectra were recorded at $25^{\circ} \mathrm{C}$ on a Varian Unity INOVA $600 \mathrm{MHz}$ NMR spectrometer equipped with a triple resonance probe and $z$-axis pulsed field gradient. For urine samples, one-dimensional spectra were acquired using a onedimensional NOESY pulse sequence with water suppression during the relaxation delay of 2 seconds, and the mixing time of $120 \mathrm{~ms}$. Sixty-four free induction decays were collected into $32 \mathrm{~K}$ data points with a spectral width of $10000 \mathrm{~Hz}$, an acquisition time of 1.64 seconds and a total pulse recycle delay of 5.76 seconds. The free induction decay (FID) was zerofilled to $64 \mathrm{~K}$ and multiplied by an exponential linebroadening function of $0.3 \mathrm{~Hz}$ prior to Fourier transformation. Resonances assignments were performed based on the extant literatures [13] and 2D ${ }^{1} \mathrm{H}^{-1} \mathrm{H}$ COSY and TOCSY spectra (data not shown).

\subsection{Multivariate Pattern Recognition Analysis of ${ }^{1} \mathrm{H} N \mathrm{NM}$} Spectra. In order to exploit all the metabolic information embedded in the spectra, all NMR spectra were data reduced to 241 integrated regions of equal width of $0.04 \mathrm{ppm}$ (buckets) corresponding to the region of $\delta 10.0$ to 0.4 using the VNMR 6.1C software package (Varian, Inc) prior to statistical analysis. Each segment consisted of the integral of the associated NMR region except the region of $\delta 6.2-$ 4.6 (containing the residual water and urine resonance) that was set to zero integral in the analysis. Following a preliminary PCA analysis, regions including $\delta 5.2, \delta 4.6, \delta$ $3.4-3.9$, and $\delta 3.2$ of all spectra were also removed due to the presence of conspicuous glucose metabolite resonances. The remaining spectral segments for each NMR spectrum were normalized to the total sum of the spectral intensity to partially compensate for differences in concentration of the samples.

The normalized integral values were then subjected to multivariate analysis as variables for the multivariate 


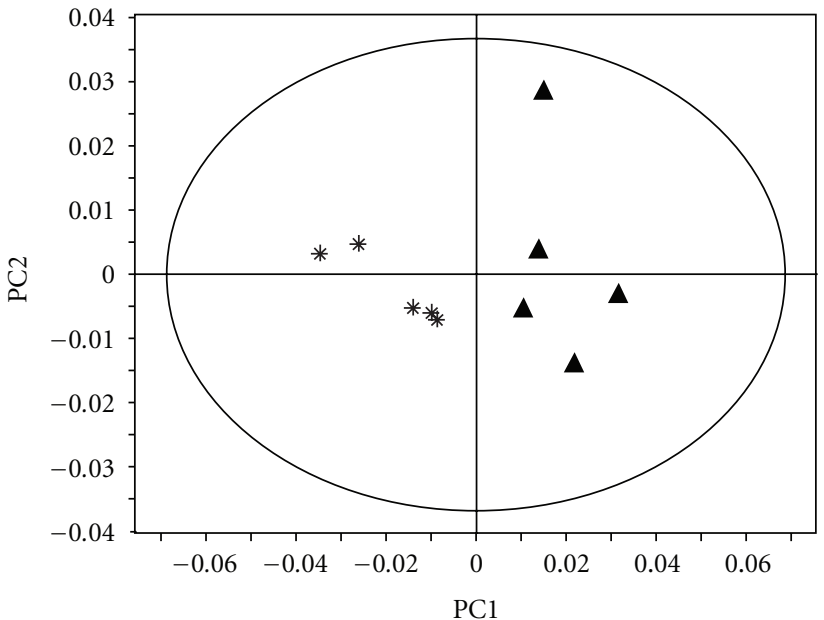

(a)

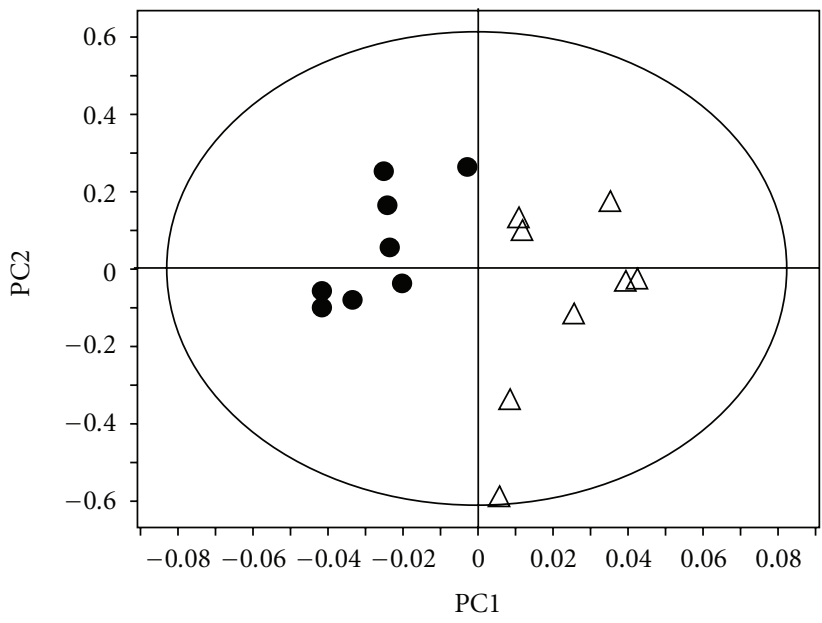

(c)

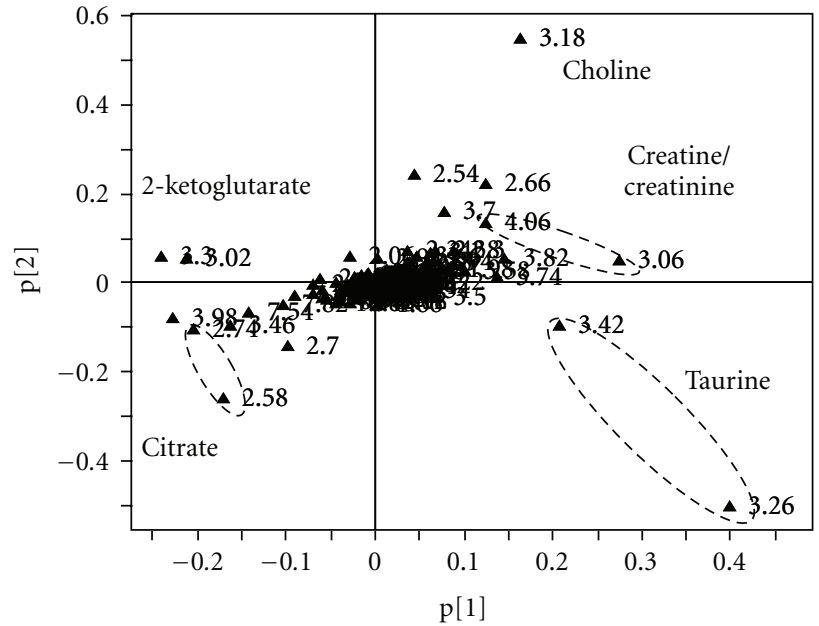

(b)

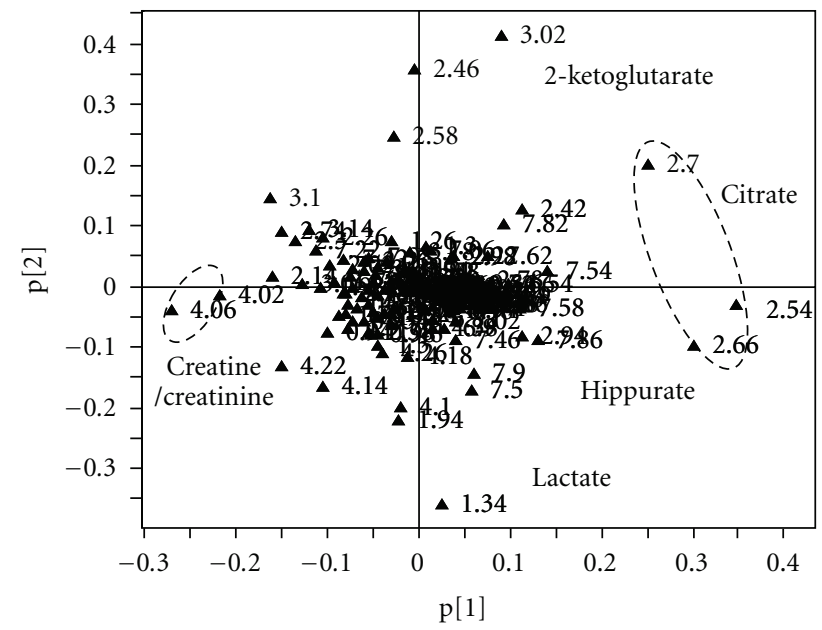

(d)

Figure 2: (a)-(b) PCA scores plot (PC1/PC2) and corresponding loading plot based on the ${ }^{1} \mathrm{H}$ NMR spectra of urine samples from Zucker lean rats $(*)$ and Zucker obese rats $(\boldsymbol{\Delta})$, respectively. (c)-(d) PCA scores plot (PC1/PC2) and corresponding loading plot based on the ${ }^{1} \mathrm{H}$ NMR spectra of urine samples from Wistar rats $(\triangle)$ and GK rats $(\bullet)$, respectively.

pattern recognition analysis using version 10.5 of SIMCA$\mathrm{P}$ software package (Umetrics, Umeå, Sweden). Principal component analysis (PCA) was performed to differentiate between the groups of samples. Data were visualized with the PC scores plot of the first two or three principal components (PC1, PC2, or PC3) to provide the most efficient $2 \mathrm{D}$ or $3 \mathrm{D}$ representation of the information contained in the data set, in which each point represents an individual spectrum of a urine sample. The corresponding loading plots, in which each point represents a single NMR spectral region segment, are used to identify which spectral variables contribute to the separation of the samples on the scores plot.

Meanwhile, in order to detect significant differences between metabolic changes, unpaired $t$-test was carried out for the comparison of integral values of spectral variables. $P$ value of less than .05 was considered statistically significant.

\section{Results and Discussion}

As represented a widely used rodent model of obesity and insulin resistance, the Zucker obese rat $(f a / f a)$ is a leptin deficient stain, characterized by obesity, insulin resistance, hyperinsulinaemia, and dyslipidaemia [14]. The GK rat is a spontaneous type 2 diabetic model [15], which shows the main features of the metabolic, hormonal, and vascular disorders usually described in T2DM [16]. Here we described the application of ${ }^{1} \mathrm{H}$ NMR-based metabonomics to investigate the urinary metabolic profiles in both rat models related to T2DM.

3.1. Analysis of Metabonomic Data. Typical ${ }^{1} \mathrm{H}$ NMR spectra of urinary samples from Zucker obese and GK rats are shown in Figure 1, in which the main resonances are assigned. In order to illustrate the differences in the metabolic profile, 
TABLE 1: Summary of the changes in relative amounts of urine metabolites of different group rats indicated by ${ }^{1} \mathrm{H}$ NMR spectra $(\mathrm{mean} \pm$ $\mathrm{SD})$.

\begin{tabular}{lccccc}
\hline$\delta{ }^{1} \mathrm{H}$ & Metabolites & Zucker lean & Zucker obese & \multicolumn{2}{c}{ Wistar } \\
\hline 1.33 & Lactate & $1.67 \pm 0.12$ & $1.10 \pm 0.11^{* *}$ & $2.97 \pm 0.95$ & $1.86 \pm 0.17^{* *}$ \\
1.46 & Alanine & $0.44 \pm 0.01$ & $0.37 \pm 0.03^{* *}$ & $0.63 \pm 0.07$ & $0.66 \pm 0.04$ \\
1.93 & Acetate & $0.98 \pm 0.19$ & $1.32 \pm 0.16^{* *}$ & $1.54 \pm 0.68$ & $1.04 \pm 0.10^{*}$ \\
2.14 & Methionine & $0.73 \pm 0.07$ & $0.75 \pm 0.08$ & $0.95 \pm 0.06$ & $1.37 \pm 0.12^{* *}$ \\
2.26 & Acetoacetate & $0.72 \pm 0.17$ & $0.80 \pm 0.09^{*}$ & $0.95 \pm 0.10$ & $1.17 \pm 0.36^{* *}$ \\
2.43 & Succinate & $1.26 \pm 0.11$ & $1.11 \pm 0.15^{* *}$ & $2.03 \pm 0.34$ & $1.87 \pm 0.26$ \\
2.46 & 2-ketoglutarate & $1.22 \pm 0.27$ & $1.04 \pm 0.10^{* *}$ & $3.93 \pm 0.56$ & $3.13 \pm 0.49^{*}$ \\
$2.52,2.67$ & Citrate & $1.92 \pm 0.26$ & $1.55 \pm 0.37^{* *}$ & $2.80 \pm 0.74$ & $1.78 \pm 0.46^{* *}$ \\
2.72 & Dimethylamine & $1.23 \pm 0.58$ & $1.84 \pm 0.17^{*}$ & $1.25 \pm 0.18$ & $1.69 \pm 0.13^{* *}$ \\
3.06 & Creatine & $2.62 \pm 0.49$ & $3.52 \pm 0.25^{* *}$ & $5.16 \pm 0.33$ & $1.00 \pm 0.15$ \\
3.20 & Choline & $0.61 \pm 0.04$ & $1.44 \pm 0.91^{* *}$ & $0.82 \pm 0.35^{* *}$ \\
$3.26,3.42$ & Taurine & $2.12 \pm 0.43$ & $2.88 \pm 0.38^{* *}$ & $2.53 \pm 0.21$ & $0.99 \pm 0.10$ \\
7.34 & Phenylalanine & $0.50 \pm 0.04$ & $0.50 \pm 0.10$ & $0.51 \pm 0.04$ & $0.70 \pm 0.22^{*}$ \\
$7.55,7.64$ & Hippurate & $0.87 \pm 0.05$ & $0.23 \pm 0.05^{* *}$ & $0.98 \pm 0.22$ & $0.75 \pm 0.08^{*}$ \\
\hline
\end{tabular}

${ }^{*} P<.05$ and ${ }^{* *} P<.01$ compared with their corresponding control rats, respectively.

NMR spectra were further segmented and subjected to PCA. The glucose resonances were excluded before the PCA described for all spectra data, for the increase in urinary glucose concentration was, well documented in GK rats [11]. In the scores plot of PCA (Figure 2(a)), the Zucker obese rats are separated from the Zucker lean rats in the direction of PC1, which indicates changes of the metabolic patterns between the two groups. The contributions of different regions of the spectra to the separation are illustrated in the loading plot (Figure 2(b)). Similar to the case of Zucker obese rats, scores plot of GK and its control rats revealed the complete separation, too (Figures 2(c) and 2(d)). Then the comparison of metabolic changes of both model rats compared with respective control rats is described in Table 1.

3.2. Comparing the Metabolic Changes of Both Models. From the metabonomic data, we could find that many of metabolic changes in Zucker obese rats were consistent with the GK rats, including the decreased levels of citrate, 2ketoglurarate, lactate, hippurate, and succinate, as well as the increased levels of creatine/creatinine, dimethylamine and acetoacetate. The results indicate similar states of metabolic changes between the two stages of diabetes, which is consistent with the changes of some amino acids concentration in Zucker diabetic rats previously reported in [12].

The energy needed for the body to function requires ATP, which primarily comes from glycolysis, glucose oxidation and lipid oxidation. Under normal physiological conditions, multiple substrates can be utilized, such as glucose, amino acids, ketone bodies, and fatty acids [17]. In GK and Zucker obese rats, the decreased levels of citrate, 2-ketoglutarate, and succinate, which are intermediates of the TCA cycle localized mainly in mitochondria, are indicative of alterations in

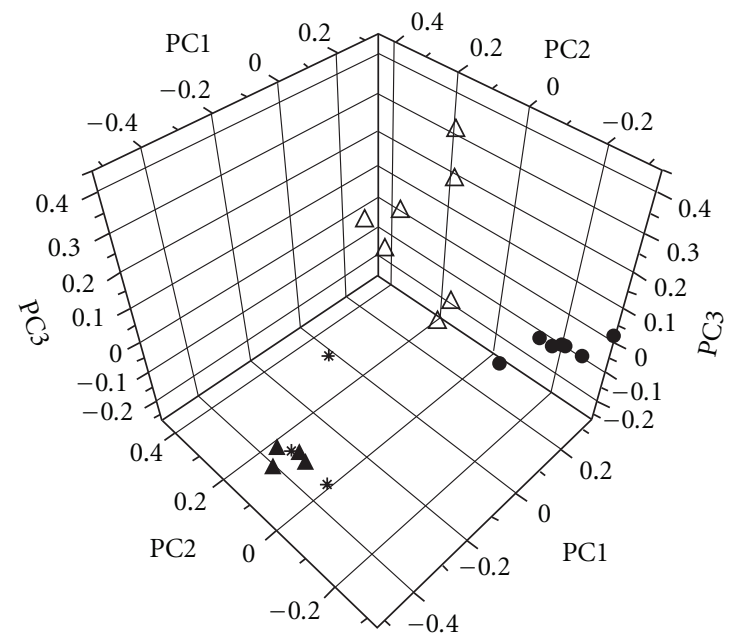

FIGURE 3: Combined analysis of the metabolomic dataset based on the ${ }^{1} \mathrm{H}$ NMR spectra of urine samples from Zucker obese rats $(\boldsymbol{\Delta})$, Zucker lean rats $(*)$, GK rats $(\bullet)$, and Wistar rats $(\triangle)$, respectively.

energy metabolism. It is known that the mitochondria produce more than $90 \%$ of energy ATP by ox-phos [18]. Serkova et al. had demonstrated the significant decrease of hepatic ATP./ADP ratio in Zucker obese rats [19], which probably implied the occurrence of impairment in mitochondrial function in diabetic state [20]. The alterations of the organic acids levels were consistent with change of related enzyme activity, such as inhibition of pyruvate kinase, glucokinase, and phosphofructokinase in the hyperglycemia state $[21,22]$. The results of both models, especially of Zucker obese rats, indicate that the mitochondrial dysfunction occurs at the whole pathogenetic process of T2DM.

From the results, we also determined the increased levels of acetoacetate, which suggests the shift in energy 
metabolism towards the formation and utilization of ketone bodies. And the increased ketone bodies production might be explained by the production of fatty acids, which were significantly increased both in Zucker obese and GK rats [23]. Also was found it the decreased level of lactate in urinary samples of both models. Our results were consistent with the GK and STZ administration for 1 month diabetic rats [24], in which they attributed the decrease of lactate to the production of ATP. The metabolic changes indicate the similarities of glucose and ketone bodies metabolism in the two stages of T2DM.

Lipid intermediary metabolites, such as choline and dimethylamine, are present in the urine, serum, and multiple tissues of mice, rats, and humans. One of the principle roles of methylamines is to act as nonperturbing renal osmolytes [25]. Our study showed that both model rats excreted higher concentrations of methylamines in the urine. The upregulation of the methylamine pathway in vivo might be linked to the osmotic compensation for raised blood glucose concentrations, or it might be indicative of a renal papillary dysfunction in diabetic state [26].

It was reported that the combined elevation of taurine and creatine levels had been regarded as biomarkers for liver damage [27]. Creatine is synthesized and metabolized in the liver, so its change of concentration reflects the alteration of liver function. Increased level of taurine in Zucker obese rats was also found in the study. Because in ${ }^{1} \mathrm{H}$ NMR spectra of urine samples, resonance peaks of taurine $(\delta 3.26$ and $\delta$ 3.42 ) overlapped with glucose, and the latter was significantly elevated in GK rats (Figure 1). As a result, we could not determine its change in GK rats. As the most abundant free amino acid in many tissues, taurine plays many physiologic and pathologic roles in vivo. Except for indication of liver dysfunction after administration of hepatotoxins [27], taurine was also a potent endogenous antioxidant, which showed protective effects against oxidative stress in diabetic rats [28]. In addition it was reported that taurine could influence blood glucose and insulin levels and play a role in the function and integrity of pancreatic $\beta$-cells, the site of insulin secretion [29].

3.3. A Combination of Two Strain Rats. A 3D-PCA scores plot of two stages of T2DM was built using the NMR data of urine samples from both model rats and their control (Figure 3). In the plot, there is distinct separation between the different samples, which indicated differences of the metabolic patterns among different groups. Interestingly, the figure shows that Zucker obese and lean rats almost lay in the same quadrant, as well as GK and Wistar rats lay in another, which indicated the similar metabolic pattern in the similar strain of rats. From the results, we could draw the conclusion that the metabolic changes caused by strain-related variation were larger than pathological state.

By comparison of two model rats, a number of metabolic alterations were identified in GK rats: (1) increased levels of creatine/creatinine, 2-ketoglutarate, lactate, citrate, hippurate, acetoacetate, alanine, and succinate; (2) decreased levels of trimethylamine, acetate, and choline compared with
Zucker obese rats. The increased concentration of energy metabolites, such as TCA intermediates (2-ketoglutarate, succinate, and citrate), lactate, and acetoacetate, reflected energy metabolic changes produced by hyperglycemia stage more than in insulin resistance stage. The results indicated in the state of hyperglycemia that the glucose and energy metabolic changes were significantly active than those in the state of insulin resistance.

Other perturbations of interest included increased methylamines and acetate metabolites in Zucker obese rats. The reason of elevation of the metabolites might be the lipid metabolic activity in Zucker obese rats more than in GK rats. In addition, the elevation of acetate level itself may contribute to impaire in renal function [9]. Compared with the GK rats, reduced urinary creatinine was shown in Zucker obese rats, which reflected altered renal tubular function in the rats [30]. These results indicated that, compared with GK rats, Zucker obese rats might be more inclined to develop diabetic nephropathy. However, more data are required to support this hypothesis.

\section{Conclusion}

To our knowledge this work firstly compared metabolic levels in the two stages of pre- and diabetes by metabonomic technology. Many of the changes observed in insulin resistance stage were similar to those found in hyperglycemia stage. However, a combination of the urine samples of all the rats indicated that the metabolic changes caused by strainrelated variation were larger than those in pathological state. In addition, compared with Zucker obese rats the, GK rats had enhanced energy metabolic pathways, which indicated energy metabolic changes produced in hyperglycemia stage more than in insulin resistance stage. The results will be of potential benefit to understanding the pathogenic process of T2DM and offers important information for the diagnosis and treatment of the disease.

\section{Acknowledgments}

This work was supported by the National Natural Science Foundation of China (20705037 and 30730026), the Medicine \& Health Special Foundation of Zhejiang Province (2010QNA016), and the National Science and Technology Major Project "Key New Drug Creation and Manufacturing Program” (2009ZX09301-001).

\section{References}

[1] A. A. Ismail and G. V. Gill, "The epidemiology of type 2 diabetes and its current measurement," Bailliere's Best Practice and Research, vol. 13, no. 2, pp. 197-220, 1999.

[2] T. Huo, S. Cai, X. Lu, Y. Sha, M. Yu, and F. Li, "Metabonomic study of biochemical changes in the serum of type 2 diabetes mellitus patients after the treatment of metformin hydrochloride," Journal of Pharmaceutical and Biomedical Analysis, vol. 49, no. 4, pp. 976-982, 2009.

[3] D. L. Rothman, I. Magnusson, G. Cline et al., "Decreased muscle glucose transport/phosphorylation is an early defect in 
the pathogenesis of non-insulin-dependent diabetes mellitus," Proceedings of the National Academy of Sciences of the United States of America, vol. 92, no. 4, pp. 983-987, 1995.

[4] G. W. Cline, K. F. Petersen, M. Krssak et al., "Impaired glucose transport as a cause of decreased insulin-stimulated muscle glycogen synthesis in type 2 diabetes," New England Journal of Medicine, vol. 341, no. 4, pp. 240-246, 1999.

[5] J. K. Nicholson, J. Connelly, J. C. Lindon, and E. Holmes, "Metabonomics: a platform for studying drug toxicity and gene function," Nature Reviews Drug Discovery, vol. 1, no. 2, pp. 153-161, 2002.

[6] J. L. Griffin and A. Vidal-Puig, "Current challenges in metabolomics for diabetes research: a vital functional genomic tool or just a ploy for gaining funding?" Physiological Genomics, vol. 34, no. 1, pp. 1-5, 2008.

[7] R. Williams, E. M. Lenz, A. J. Wilson et al., "A multianalytical platform approach to the metabonomic analysis of plasma from normal and zucker (fa/fa) obese rats," Molecular BioSystems, vol. 2, no. 3-4, pp. 174-183, 2006.

[8] H. J. Major, R. Williams, A. J. Wilson, and I. D. Wilson, "A metabonomic analysis of plasma from Zucker rat strains using gas chromatography/mass spectrometry and pattern recognition," Rapid Communications in Mass Spectrometry, vol. 20, no. 22, pp. 3295-3302, 2006.

[9] R. E. Williams, E. M. Lenz, M. Rantalainen, and I. D. Wilson, "The comparative metabonomics of age-related changes in the urinary composition of male Wistar-derived and Zucker (fa/fa) obese rats," Molecular BioSystems, vol. 2, no. 3-4, pp. 193-202, 2006.

[10] R. E. Williams, E. M. Lenz, J. A. Evans et al., "A combined 1H NMR and HPLC-MS-based metabonomic study of urine from obese (fa/fa) Zucker and normal Wistar-derived rats," Journal of Pharmaceutical and Biomedical Analysis, vol. 38, no. 3, pp. 465-471, 2005.

[11] R. M. Salek, M. L. Maguire, E. Bentley et al., "A metabolomic comparison of urinary changes in type 2 diabetes in mouse, rat, and human," Physiological Genomics, vol. 29, no. 2, pp. 99 108, 2007.

[12] E. P. Wijekoon, C. Skinner, M. E. Brosnan, and J. T. Brosnan, "Amino acid metabolism in the Zucker diabetic fatty rat: effects of insulin resistance and of type 2 diabetes," Canadian Journal of Physiology and Pharmacology, vol. 82, no. 7, pp. 506514, 2004.

[13] Q. Wang, Y. Jiang, C. Wu et al., "Study of a novel indolin2-ketone compound Z24 induced hepatotoxicity by NMRspectroscopy-based metabonomics of rat urine, blood plasma, and liver extracts," Toxicology and Applied Pharmacology, vol. 215, no. 1, pp. 71-82, 2006.

[14] H. G. Gika, G. A. Theodoridis, and I. D. Wilson, "Hydrophilic interaction and reversed-phase ultra-performance liquid chromatography TOF-MS for metabonomic analysis of Zucker rat urine," Journal of Separation Science, vol. 31, no. 9, pp. 1598-1608, 2008.

[15] J. Galli, L.-S. Li, A. Glaser et al., "Genetic analysis of noninsulin dependent diabetes mellitus in the GK rat," Nature Genetics, vol. 12, no. 1, pp. 31-37, 1996.

[16] M. Gotoh, C. Li, M. Yatoh, N. Okabayashi, S. Habu, and Y. Hirooka, "Hypothalamic monoamine metabolism is different between the diabetic GK (Goto-Kakizaki) rats and streptozotocin-induced diabetic rats," Brain Research, vol. 1073-1074, no. 1, pp. 497-501, 2006.

[17] L. Fritsche, C. Weigert, H.-U. Häring, and R. Lehmann, "How insulin receptor substrate proteins regulate the metabolic capacity of the liver-implications for health and disease," Current Medicinal Chemistry, vol. 15, no. 13, pp. 1316-1329, 2008.

[18] S. R. Pieczenik and J. Neustadt, "Mitochondrial dysfunction and molecular pathways of disease," Experimental and Molecular Pathology, vol. 83, no. 1, pp. 84-92, 2007.

[19] N. J. Serkova, M. Jackman, J. L. Brown et al., "Metabolic profiling of livers and blood from obese Zucker rats," Journal of Hepatology, vol. 44, no. 5, pp. 956-962, 2006.

[20] W. -J. Deng, S. Nie, J. Dai, J. -R. Wu, and R. Zeng, "Proteome, phosphoproteome, and hydroxyproteome of liver mitochondria in diabetic rats at early pathogenic stages," Molecular and Cellular Proteomics, vol. 9, no. 1, pp. 100-116, 2010.

[21] V. Vats, S. P. Yadav, and J. K. Grover, "Effect of T. foenumgraecum on glycogen content of tissues and the key enzymes of carbohydrate metabolism," Journal of Ethnopharmacology, vol. 85, no. 2-3, pp. 237-242, 2003.

[22] F. Belfiore, S. Iannello, R. Campione, and G. Volpicelli, "Metabolic effects of high glucose concentrations: inhibition of hepatic pyruvate kinase," Diabetes Research, vol. 10, no. 4, pp. 183-186, 1989.

[23] M. J. Azain and J. A. Ontko, "Ketone body utilization for lipogenesis in the perfused liver of the obese Zucker rat," Hormone and Metabolic Research, vol. 22, no. 11, pp. 561-565, 1990.

[24] S. Amaral, A. J. Moreno, M. S. Santos, R. Seiça, and J. Ramalho-Santos, "Effects of hyperglycemia on sperm and testicular cells of Goto-Kakizaki and streptozotocin-treated rat models for diabetes," Theriogenology, vol. 66, no. 9, pp. 20562067, 2006.

[25] C. L. Gavaghan, E. Holmes, E. Lenz, I. D. Wilson, and J. K. Nicholson, "An NMR-based metabonomic approach to investigate the biochemical consequences of genetic strain differences: application to the C57BL10J and Alpk:ApfCD mouse," FEBS Letters, vol. 484, no. 3, pp. 169-174, 2000.

[26] I. Messana, F. Forni, F. Ferrari, C. Rossi, B. Giardina, and C. Zuppi, "Proton nuclear magnetic resonance spectral profiles of urine in type II diabetic patients," Clinical Chemistry, vol. 44, no. 7, pp. 1529-1534, 1998.

[27] N. J. Waters, C. J. Waterfield, R. D. Farrant, E. Holmes, and J. K. Nicholson, "Metabonomic deconvolution of embedded toxicity: application to thioacetamide hepato- and nephrotoxicity," Chemical Research in Toxicology, vol. 18, no. 4, pp. 639654, 2005.

[28] L.-J. Wang, Y.-H. Yu, L.-G. Zhang et al., "Taurine rescues vascular endothelial dysfunction in streptozocin-induced diabetic rats: correlated with downregulation of LOX-1 and ICAM-1 expression on aortas," European Journal of Pharmacology, vol. 597, no. 1-3, pp. 75-80, 2008.

[29] E. C. Kulakowski and J. Maturo, "Hypoglycemic properties of taurine: not mediated by enhanced insulin release," Biochemical Pharmacology, vol. 33, no. 18, pp. 2835-2838, 1984.

[30] N. C. Turner, P. J. Morgan, A. C. Haynes, M. Vidgeon-Hart, N. Toseland, and J. C. Clapham, "Elevated renal endothelin1 clearance and mRNA levels associated with albuminuria and nephropathy in non insulin-dependent diabetes mellitus: studies in obese fa/fa Zucker rats," Clinical Science, vol. 93, no. 6, pp. 565-571, 1997. 


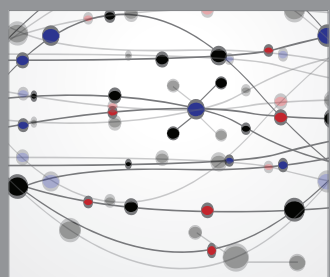

The Scientific World Journal
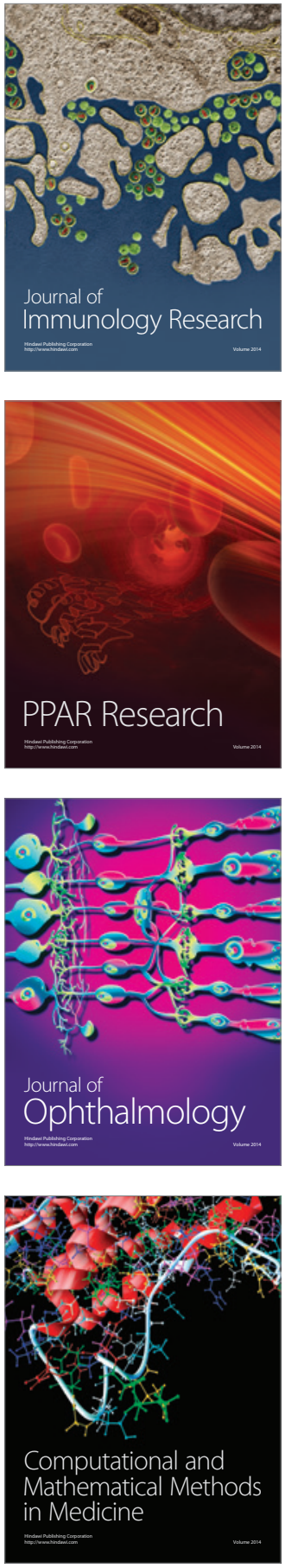

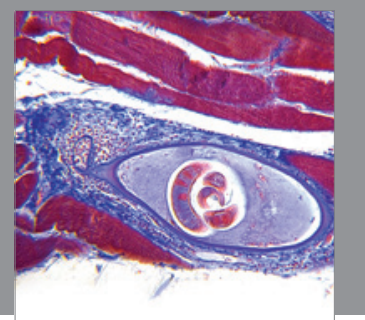

Gastroenterology

Research and Practice
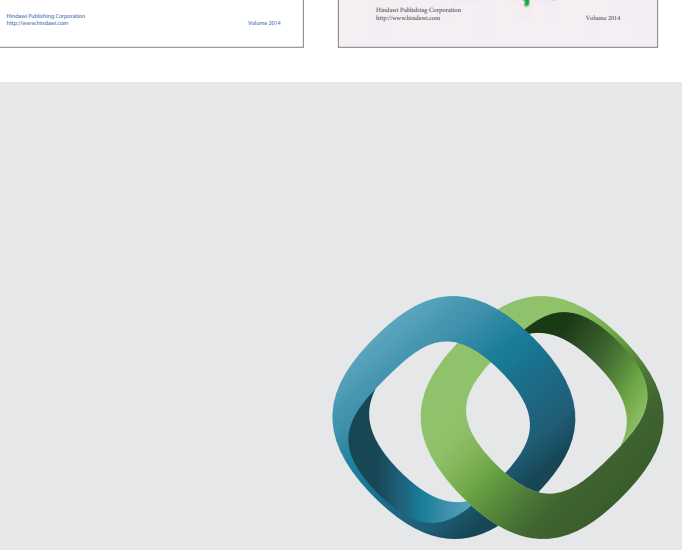

\section{Hindawi}

Submit your manuscripts at

http://www.hindawi.com
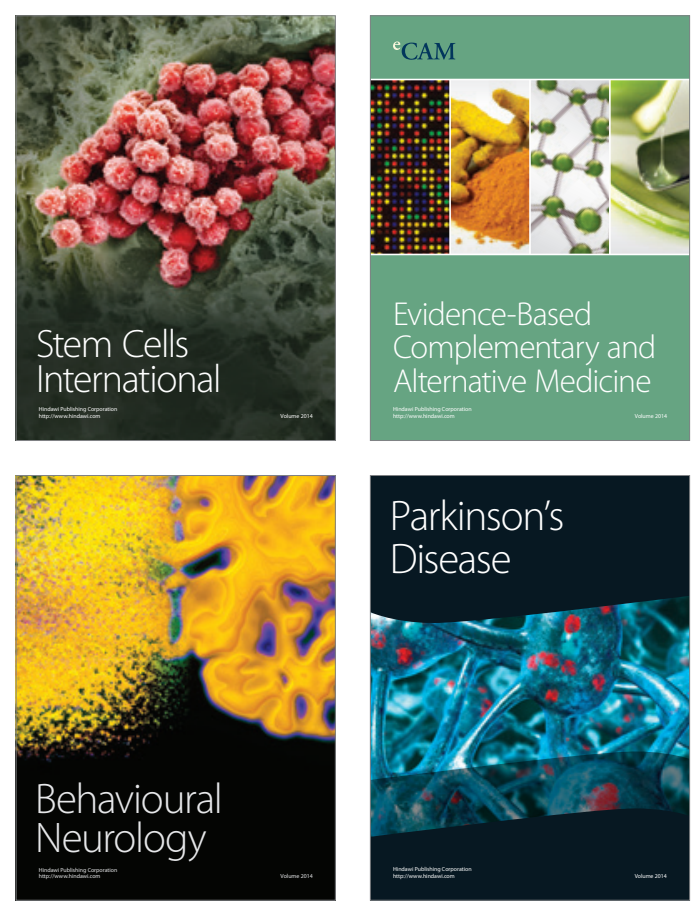

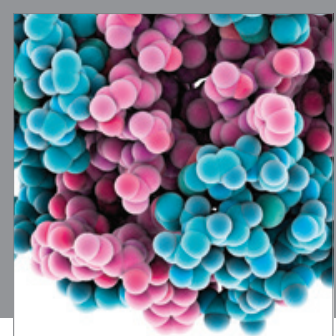

Journal of
Diabetes Research

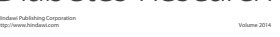

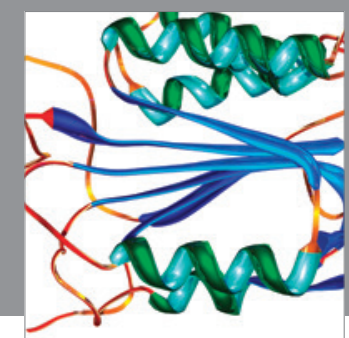

Disease Markers
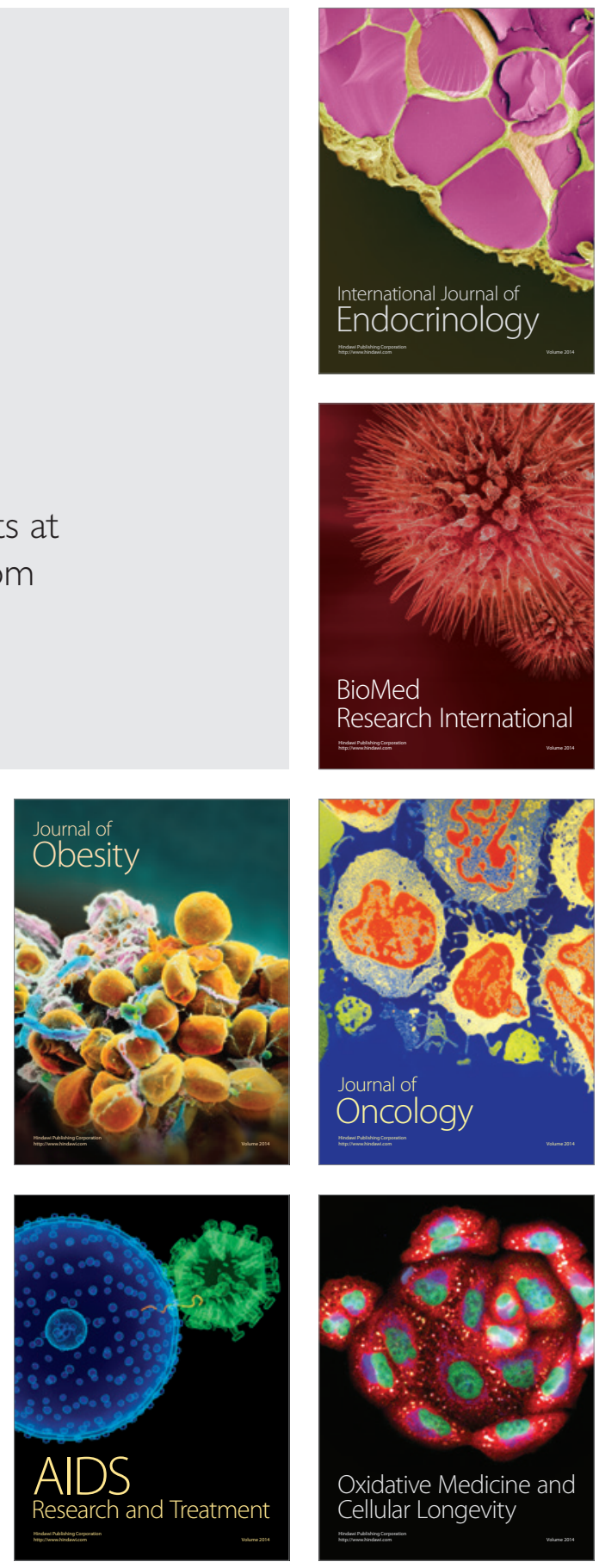